\title{
Research on Synergetic Evolution of container transportion
}

\author{
Liqun Ding ${ }^{1, a}$ \\ ${ }^{1}$ Transportation planning and management, Wuhan University of Technology, Wuhan Technical \\ College of Communication, Wuhan ,430065, China \\ a519972556@qq.com
}

Keywords: container transportion system elements co evolution;

Abstract:According to the theory of collaborative research, pointed out that the overall function of the container transport system is not a single function, but by rearranging the elements of the system, achieve the reasonable allocation of resources. Through the co evolution equation, in-depth analysis of container transportion system between each element of the system, cooperative operation of system development and the importance of collaborative process, has important reference value for the optimization of container transportion.

\section{Introduction}

With the globalization of international trade, the operation level and service capacity of international logistics as a derivative demand are facing greater challenges. The port is no longer the end of the transportation line, but a node in the international logistics chain, which is one of the set of container transit station. The goal of international transport service has been changed from "port to port" to "door to door". Therefore, the integrated transportation concept is generally recognized. the rapid development of international multimodal transport made the result of continuous extension of transport routes, so container transport routes gradually increased.

At present, the global railway container transport volume accounted for the proportion of total rail transport of goods is about $20 \%$ to $40 \%$, of which the developed countries a higher proportion of rail container transport, the official release of the data show that the United States $49 \%$ France is $40 \%$, Britain $30 \%$, Germany $20 \%$. The proportion of the world's container hot metal container transport as shown in Figure 1 as follows.

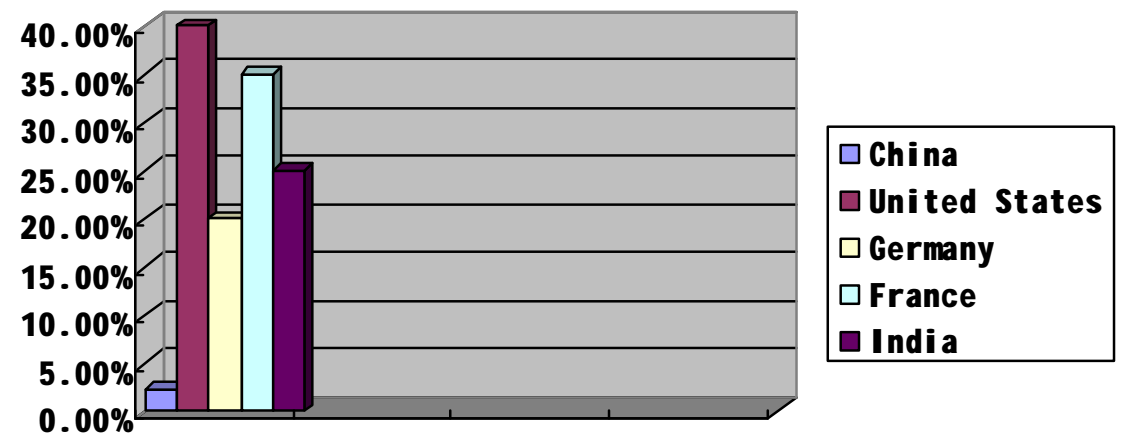

Figure 1: The proportion of container transport in the world

From the above data analysis shows, that China's railway freight volume growth is slow, China's railway transport management system has long-term independence, low market flexibility, large bulk cargo demand throughout, taking up most of the railway capacity, bulk cargoes are mostly productive materials, transportation, handling and warehousing requirements are relatively low, Departments tend to be the main railway line capacity is mainly allocated to the bulk of bulk, to suppress the development of railway container transport. and the completion of the main cargo volume from bulk cargo, container rail transport accounted for a small proportion, and Load capacity is small, and the ship does not match the load. 


\section{Container transportion coordination mechanism}

According to the synergetic theory, the coordination of the system and its elements and subsystems in the process of operation leads to a new generation of cooperative structures and functions. Synergetic motion between subsystems will drive the system toward two opposite directions of evolution: one is from the orderly state of spontaneous to disordered state changes, the other is from the disorder to the orderly process of evolution.

Through the development of the whole society and economy, the coverage and inter-regional connection of the transportation lines, the standardization and rationalization of the transportation organization and management, the operation of the transportation industry and related industries, the professional skills of the employees and the improvement of professional accomplishment. Coordination is the basis for the establishment and development of cooperative relationships among transport enterprises in multimodal transport.

In addition, the container transportation time fluctuates greatly, the container cargo is more in the east, less in the south and more in the north. A series of imbalances lead to the difficulty in the supply organization at both ends of the transportation and the sharp contradiction between the mixed goods and passengers, which leads to the restrictive transportation of the railways, Increasing the difficulty of empty container transport, a serious impediment to the railway market operations and multimodal transport development.

In order to achieve door-to-door service, Container transportion is divided into several sub-systems, according to the mode of transport can be divided into public-rail transport, public-water transport, Container transportion, taking into account the matching of transport and facelift cost, under the premise of advocating the selection of Container transportion .

The synergetic theory describes the equilibrium state as the system state parameter, which does not change with time, and will not appear in the phenomenon of macro-transmission. Unstable equilibrium has two characteristics: First, as time goes on, the system state parameters and space no longer changes; Second, there is no macro-physical flow system, such as particle flow.

\section{Co-evolution equation and distribution of Container transportion}

China's rapid economic development, grain, large-scale production equipment, coal, oil and other productive materials occupy large railway capacity, container transport only a small share of the railway sector tend to the main railway line capacity is mainly allocated to bulk cargo, To suppress the development of container transport.

For example, the most important transport hub in the middle reaches of the Yangtze River - Wuhan, with the conditions of molten iron transport, the use of the Yangtze River waterway unique advantages, through the upper reaches of the Yangtze River in Chongqing to the lower reaches of the Yangtze River in Nanjing, Shanghai inland routes, As a central city in the central part of China, it is an important transportation hub and distribution center in the central region. The transportation infrastructure is better, the coverage of railway and highway lines is higher. Through the vertical and horizontal transportation of the roads, it can meet the increasing freight of the surrounding area Quantity demand.

Therefore, the main outstanding contradictions of the tightly grasp Container transportion system, whicn are planned from the top design.It is necessary to consider the system to containing and promoting the relationship between the various elements of the system , and to promote the generation of collaborative behavior which is essential.

Through the cooperative evolution equation, it is possible to grasp the collaborative process between the elements of the container transportation.

$$
\S=N(q, \alpha)+F
$$

Each parameter is expressed:

$$
q--- \text { Order parameter }
$$




\section{$\alpha---$ Control parameters \\ $F---$ Random fluctuation force \\ $N---$ Non - linear function vector driving force}

The evolution model is composed of system order parameters $q$ and different order derivative equations. This paper will study the evolution mechanism of the container transportation system by evolutionary model.

In the container iron and water transport collection and distribution system, set the driving force $N$ for the maximum system efficiency, set $X(t)$ the system efficiency, random fluctuation force $F=0$, that is

$$
\frac{d X(t)}{d t}=k X\left(1-\frac{X}{N}\right)
$$

Each parameter is expressed:

$k---$ Coefficient of synergy benefit

$X---$ The growth factor over time

$$
\begin{aligned}
& \frac{X}{N} \text { Benefit Growth Acceleration } \\
& 1-\frac{X}{N} \text { A reduction factor with time }
\end{aligned}
$$

Equation (2) shows that the container transport collection and distribution system is non-linear. When $k>0, N>0$, The solution of (2) is

$$
X=\frac{N}{1+C \exp \left(-\frac{k}{N} t\right)}
$$

Based on the above, the synergistic mechanism of Container transport intermodal collection and distribution system,

Assume that the Container railway and waterway combined transport intermodal collection and distribution system is composed of the railway sector $C_{1}$, the owner $C_{2}$, port enterprise ${ }^{C_{3}} \mathrm{~s}$. According to the evolution equation:

$$
\left\{\begin{array}{l}
\frac{d X_{1}(t)}{d t}=k_{1} X_{1}\left(1-\frac{X_{1}}{N}\right) \\
\frac{d X_{2}(t)}{d t}=k_{2} X_{2}\left(1-\frac{X_{2}}{N}\right) \\
\frac{d X_{3}(t)}{d t}=k_{3} X_{3}\left(1-\frac{X_{3}}{N}\right)
\end{array}\right.
$$

Let $\lambda_{1}$ be the contribution of $C_{1}$ to $C_{2} 、 C_{3}$, let $\lambda_{2}$ be the contribution of $C_{2}$ to $C_{1} 、 C_{3}$, and $\lambda_{3}$ be the contribution of $C_{3}$ to $C_{1}, C_{2}$.

After the establishment of an effective relationship between the three can be through the sharing of transport resources and transport operations of the collaborative effect of the Container railway and waterway combined transport system set to the orderly development of the distribution system.

$\left.\left.\left.\lambda_{1}\right\rangle 0, \lambda_{2}\right\rangle 0, \lambda_{3}\right\rangle 0$, 且 $\lambda_{1}+\lambda_{2}\left\langle 1, \lambda_{, 1}+\lambda_{3}\left\langle 1, \lambda_{, 2}+\lambda_{3}\langle 1 \quad\right.\right.$, Given the co-evolution equation: 


$$
\left\{\begin{array}{l}
\frac{d X_{1}(t)}{d t}=k_{1} X_{1}\left(1-\frac{X_{1}}{N_{1}}+\lambda_{1} \frac{X_{2}}{N_{2}}+\lambda_{1} \frac{X_{3}}{N_{3}}\right. \\
\frac{d X_{2}(t)}{d t}=k_{2} X_{2}\left(1-\frac{X_{2}}{N_{2}}+\lambda_{2} \frac{X_{1}}{N_{1}}+\lambda_{2} \frac{X_{3}}{N_{3}}\right. \\
\frac{d X_{3}(t)}{d t}=k_{3} X_{3}\left(1-\frac{X_{3}}{N_{3}}+\lambda_{3} \frac{X_{1}}{N_{1}}+\lambda_{3} \frac{X_{2}}{X_{2}}\right.
\end{array}\right)
$$

When the synergistic state of the Container railway and waterway combined transport system is stable, it can be expressed as.

$$
\left\{\begin{array}{l}
\frac{d X_{1}(t)}{d t}=k_{1} X_{1}\left(1-\frac{X_{1}}{N_{1}}+\lambda_{1} \frac{X_{2}}{N_{2}}+\lambda_{1} \frac{X_{3}}{N_{3}}\right)=0 \\
\frac{d X_{2}(t)}{d t}=k_{2} X_{2}\left(1-\frac{X_{2}}{N_{2}}+\lambda_{2} \frac{X_{1}}{N_{1}}+\lambda_{2} \frac{X_{3}}{N_{3}}\right)=0 \\
\frac{d X_{3}(t)}{d t}=k_{3} X_{3}\left(1-\frac{X_{3}}{N_{3}}+\lambda_{3} \frac{X_{1}}{N_{1}}+\lambda_{3} \frac{X_{2}}{X_{2}}\right)=0
\end{array}\right.
$$

Solution of the equation, eight equilibrium points,

$$
\begin{aligned}
& A_{1}(0,0,0), A_{2}\left(N_{1}, 0,0\right), A_{3}\left(0, N_{2}, 0\right), A_{4}\left(0,0, N_{3}\right), A_{5}\left(0,-\frac{N_{2}\left(1+\lambda_{2}\right)}{\lambda_{2} \lambda_{3}-1},-\frac{N_{3}\left(1+\lambda_{3}\right)}{\lambda_{2} \lambda_{3}-1}\right), \\
& A_{6}\left(-\frac{N_{1}\left(1+\lambda_{1}\right)}{\lambda_{1} \lambda_{3}-1}, 0,-\frac{N_{3}\left(1+\lambda_{3}\right)}{\lambda_{1} \lambda_{3}-1}\right), A_{7}\left(-\frac{N_{1}\left(1+\lambda_{1}\right)}{\lambda_{1} \lambda_{2}-1},-\frac{N_{2}\left(1+\lambda_{2}\right)}{\lambda_{1} \lambda_{2}-1}, 0\right), \\
& A_{8}\left(\begin{array}{l}
\left.\frac{N_{1}\left(\lambda_{2} \lambda_{3}-1\right)-N_{1} \lambda_{1}\left(\lambda_{2}+\lambda_{3}+2\right)}{\lambda_{1} \lambda_{2}+\lambda_{1} \lambda_{3}+\lambda_{2} \lambda_{3}+2 \lambda_{1} \lambda_{2} \lambda_{3}-1}, \frac{N_{2}\left(\lambda_{1} \lambda_{3}-1\right)-N_{2} \lambda_{2}\left(\lambda_{1}+\lambda_{3}+2\right)}{\lambda_{1} \lambda_{2}+\lambda_{1} \lambda_{3}+\lambda_{2} \lambda_{3}+2 \lambda_{1} \lambda_{2} \lambda_{3}-1},\right) \\
\frac{N_{3}\left(\lambda_{1} \lambda_{2}-1\right)-N_{3} \lambda_{3}\left(\lambda_{1}+\lambda_{2}+2\right)}{\lambda_{1} \lambda_{2}+\lambda_{1} \lambda_{3}+\lambda_{2} \lambda_{3}+2 \lambda_{1} \lambda_{2} \lambda_{3}-1}
\end{array}\right)
\end{aligned}
$$

The

obtained eight equilibrium points $A\left(X_{1}^{*}, X_{2}^{*}, X_{3}^{*}\right)$ are expanded in Taylor series, and omitting the second and the above terms, we can get the equation group, the coefficient matrix of this system is

$$
\begin{aligned}
& {\left[k_{1}\left(1-\frac{2 X_{1}}{N_{1}}+\frac{\lambda_{1} X_{2}}{N_{2}}+\frac{\lambda_{1} X_{3}}{N_{3}}\right)\right.} \\
& \frac{k_{1} \lambda_{1} X_{1}}{N_{2}} \\
& \frac{k_{1} \lambda_{1} X_{1}}{N_{3}} \\
& \frac{k_{2} \lambda_{2} X_{2}}{N_{1}} \\
& k_{2}\left(1-\frac{2 X_{2}}{N_{2}}+\frac{\lambda_{2} X_{1}}{N_{1}}+\frac{\lambda_{2} X_{3}}{N_{3}}\right) \\
& \frac{k_{2} \lambda_{2} X_{2}}{N_{3}} \\
& \frac{k_{3} \lambda_{3} X_{3}}{N_{1}} \\
& \frac{k_{3} \lambda_{3} X_{3}}{N_{2}} \\
& k_{3}\left(1-\frac{2 X_{3}}{N_{3}}+\frac{\lambda_{3} X_{1}}{N_{1}}+\frac{\lambda_{3} X_{2}}{N_{2}}\right)
\end{aligned}
$$

The value of the equilibrium point $A_{1}, A_{2}, A_{3}, A_{4}, A_{5}, A_{6}, A_{7}, A_{8}$ is substituted into the matrix of equation (7), and the eigenvalues of the matrix are obtained. If the eigenvalues are different, and are positive, indicating that the system is unstable; if the eigenvalues have positive and negative values, indicating that the system is not stable; If the eigenvalues are negative, and are not the same, that the system is in a stable state. According to the above principles, $A_{1}, A_{2}, A_{3}, A_{4}, A_{5}, A_{6}, A_{7}$ is the unstable equilibrium point, only $A_{8}$ is the stable equilibrium point.

It can be concluded that the synergistic effect between the $C_{1} 、 C_{2}, C_{3}$ Container railway and 
waterway combined transport and distribution systems in the container can be expressed as

$$
\begin{aligned}
& \frac{N_{1}\left(\lambda_{2} \lambda_{3}-1\right)-N_{1} \lambda_{1}\left(\lambda_{2}+\lambda_{3}+2\right)}{\lambda_{1} \lambda_{2}+\lambda_{1} \lambda_{3}+\lambda_{2} \lambda_{3}+2 \lambda_{1} \lambda_{2} \lambda_{3}-1} \frac{N_{2}\left(\lambda_{1} \lambda_{3}-1\right)-N_{2} \lambda_{2}\left(\lambda_{1}+\lambda_{3}+2\right)}{\lambda_{1} \lambda_{2}+\lambda_{1} \lambda_{3}+\lambda_{2} \lambda_{3}+2 \lambda_{1} \lambda_{2} \lambda_{3}-1}, \\
& \frac{N_{3}\left(\lambda_{1} \lambda_{2}-1\right)-N_{3} \lambda_{3}\left(\lambda_{1}+\lambda_{2}+2\right)}{\lambda_{1} \lambda_{2}+\lambda_{1} \lambda_{3}+\lambda_{2} \lambda_{3}+2 \lambda_{1} \lambda_{2} \lambda_{3}-1}
\end{aligned}
$$

When the system reaches the steady state, the amplitude of the fluctuation becomes smaller and the decay is faster. When the system enters the critical point, the intensity of each subsystem moves towards the average gradually, at this moment, the coupling between the various subsystems is more active. At the same time, the fluctuation of partial coupling continues to affect the system, but the subsystems lacking more responses show attenuation performance. The subsystems whose boundary conditions satisfy the fluctuation fast response continue to amplify, And then grow into the order for the drive system into the giant fluctuations, into the sequence of disposable system parameters. The above is the co-evolution mechanism of container hot metal intermodal distribution and distribution system.

\section{example analysis}

Wuhan Port is a comprehensive transportation hub in the central region, which plays an important role in the development of the regional economy. The container transportation in the harbor area is very important for the construction of multi-modal transportation, shipping resources allocation and bonded processing conditions. It is one of the key indexes to evaluate the logistics operation efficiency of the hub port,but, most of our ports are not directly connected with the railway, whether it is a collection and distribution subsystem, information system, dock berth loading and unloading subsystem or warehouse storage management subsystem, the railway station and the port area are completely independent of the operation System, therefore, a long time, the port of the collection and distribution operations mostly by road transport commitment, that is, set cards or other cargo vehicles to complete the port cargo collection and distribution.

In the process of this coordinated movement, each transport mode is a subsystem of this large system, and the system is composed of several sub-systems, and the macro-control of the government is the key to the comprehensive transportation system. Form orderly to provide a certain amount of energy, and to guide and promote the integrated transport system self-organization structure of the formation and improvement.

Set the port of Wuhan Iron and Steel Container Transport iron and water transport system set with $C_{1}$, the owner with $C_{2}$, port enterprises with $C_{3}$, set $\left.\left.\left.\lambda_{1}\right\rangle 0, \quad \lambda_{2}\right\rangle 0, \quad \lambda_{3}\right\rangle 0$, and $\lambda_{1}+\lambda_{2}\left\langle 1, \lambda_{, 1}+\lambda_{3}\left\langle 1, \quad \lambda_{, 2}+\lambda_{3}\left\langle 1\right.\right.\right.$, the railway sector as the $\lambda_{1}$ argest impact on the system, set up a $C_{1}$ for the $C_{2} 、 C_{3}$ collaborative campaign contribution, Take 0.5 , set $\lambda_{2}$ is the contribution of $C_{2}$ to the contribution of $C_{1} 、 C_{3}$, take 0.2 , set $\lambda_{3}$ is the contribution of $C_{3}$ to $C_{1} 、 C_{2}$, take 0.3 .

The contribution of the value of the value of the container iron and water transportation intermodal collection and distribution system in a stable equilibrium point $C_{1} 、 C_{2} 、 C_{3}$ the synergy between:

$$
\begin{aligned}
& \frac{N_{1}\left(\lambda_{2} \lambda_{3}-1\right)-N_{1} \lambda_{1}\left(\lambda_{2}+\lambda_{3}+2\right)}{\lambda_{1} \lambda_{2}+\lambda_{1} \lambda_{3}+\lambda_{2} \lambda_{3}+2 \lambda_{1} \lambda_{2} \lambda_{3}-1} \frac{N_{2}\left(\lambda_{1} \lambda_{3}-1\right)-N_{2} \lambda_{2}\left(\lambda_{1}+\lambda_{3}+2\right)}{\lambda_{1} \lambda_{2}+\lambda_{1} \lambda_{3}+\lambda_{2} \lambda_{3}+2 \lambda_{1} \lambda_{2} \lambda_{3}-1}, \\
& \frac{N_{3}\left(\lambda_{1} \lambda_{2}-1\right)-N_{3} \lambda_{3}\left(\lambda_{1}+\lambda_{2}+2\right)}{\lambda_{1} \lambda_{2}+\lambda_{1} \lambda_{3}+\lambda_{2} \lambda_{3}+2 \lambda_{1} \lambda_{2} \lambda_{3}-1}
\end{aligned}
$$

The synergistic benefit among $C_{1} 、 C_{2} 、 C_{3}$ is obtained, 


$$
C_{1}=3.48 N_{1}, C_{2}=2.24 N_{2}, C_{3}=2.71 N_{3} \text {, }
$$

The nonlinear driving force of vector $N$ is the maximum system benefit, thus the railway department, the owner and the port enterprise are positively related to each other. And also with the driving force elements are also a positive correlation.

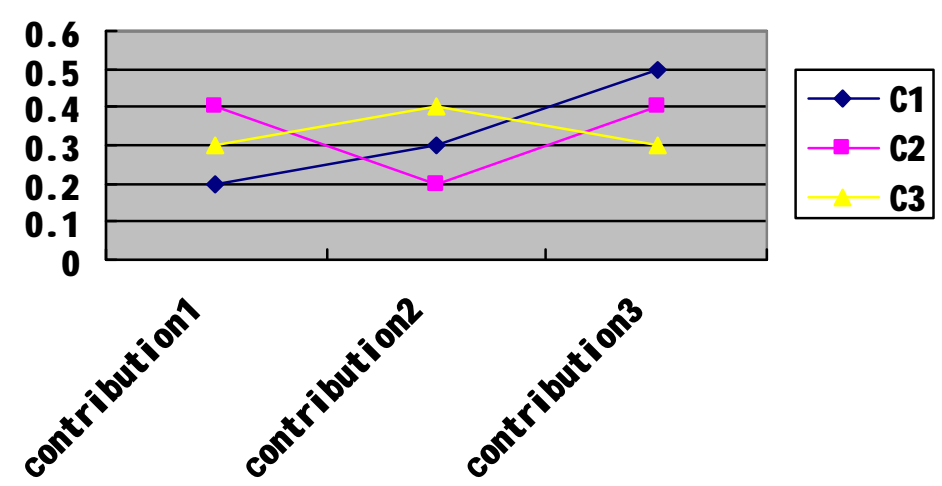

Figure2: the coordinated operation relationship of the Wuhan port Container transport system

Therefore, to meet the transport total cost minimum, the shortest transportation time, transport quality optimal transport requirements, we must make the coordinated operation of various modes of transport, to achieve the overall objective of integrated transport optimal, the coordination of the system and its elements and subsystems in the process of operation leads to a new generation of cooperative structures and functions. in this process, integrated transport coordinated development And synergetic theory describes the phenomenon of close ties.

\section{Conclusion}

According to the actual level of operation, scheduling practical ability design public information platform, and continuously improve. Through the co evolution equation, in-depth analysis of container transportion system between each element of the system, cooperative operation of system development and the importance of collaborative process, has important reference value for the optimization of container transportion.In the process of implementation, special facilities, equipment, tools, management methods and systems of Container railway and waterway combined transport should be integrated design. the contain transportion can strengthen the container interoperability and reach the entire transport chain or transport network services integration.

\section{Acknowledgement}

Scientific research project of Hubei vocational and Technical Education Society (ZJGB201559): Research on the connection of Civil Engineering Experimental Teaching in Transportation Vocational and Technical Colleges.

\section{References}

[1] Fu Huimin. Transportation Corridor Railway System Coordinated Development Research [D]. Xi'an: Chang'an University, 2006

[2] Zhang Shengrui, Yan Baojie. Theoretical Analysis of Coordinated Development of Integrated Transportation System [J].

[3] Zhang Guowu. On the planning, coordination and development of transportation systems [J]. Journal of Transportation Systems Information and Engineering, 2005,5 (1): 16-24. 
[4] Janic M. An assessment of the performance of the European long intermodal freight trains(LIFTS)[J]. Transportation Research Part A: Policy and Practice, 2008, 42(10):1326-1339.

[5] Bert Van Wee, Nam Seok Kim. The relative importance of factors that influence the break-even distance of intermodal freight transport systems[J]. Journal of Transport Geography, 2011, 19(4): 859-875. 